\title{
Review of: "Privacy nudges for disclosure of personal information: A systematic literature review and meta- analysis"
}

Elisavet Andrikopoulou ${ }^{1}$

1 University of Portsmouth

Potential competing interests: The author(s) declared that no potential competing interests exist.

“However, empirical studies on digital nudging have yielded divergent results: while some studies found nudging to be highly effective, other studies found no such effects" I think you can rephrase this in a more concise way. Consider: However, some studies found nudging to be highly effective whereas other studies found no such effects.

"there is an urgent need for a systematic review" Why urgent? You do not specify any time sensitive matters in previous paragraphs.

Why is $60 \%$ the cut-off point of your included papers selection? How did you decide that? Johanna Briggs institute for example considers anything above $70 \%$ a good quality study. 